\title{
ANÁLISE DE DIFERENTES ESTUDOS EPIDEMIOLÓGICOS EM AUDIOLOGIA REALIZADOS NO BRASIL
}

\author{
Literature review on epidemiological studies conducted \\ in Audiology in Brazil
}

\author{
Aline Megumi Arakawa ${ }^{(1)}$, Érica Ibelli Sitta ${ }^{(2)}$, Magali de Lourdes Caldana ${ }^{(3)}$, \\ Sílvia Helena de Carvalho Sales-Peres ${ }^{(4)}$
}

\begin{abstract}
RESUMO
Tema: a audição, responsável pela aquisição e desenvolvimento da linguagem, é um dos sentidos que permitem a ocorrência das relações interpessoais e com o meio ambiente. Desta forma, o levantamento epidemiológico da prevalência de deficientes auditivos em uma comunidade é de extrema importância para a adequação das medidas de saúde pública nos vários níveis de prevenção. Objetivo: verificar na literatura científica, estudos que tiveram por interesse a busca de conhecimento no âmbito epidemiológico relacionados à perda auditiva no Brasil. Foram utilizados um total de 13 artigos sendo, 11 de estudos transversais, um estudo caso-controle, e outro estudo de coorte. Conclusão: Os trabalhadores expostos a ruído ocupacional têm recebido maior atenção por parte dos estudos epidemiológicos, diferentemente da população idosa e neonatal. Apenas um estudo com base populacional, seguindo o Protocolo sugerido pela OMS, foi realizado. É importante a realização de mais estudos relacionados à deficiência auditiva no país a fim de elaborar ações de saúde e assistência adequadas às necessidades locais.
\end{abstract}

DESCRITORES: Audiologia; Epidemiologia; Perda Auditiva; Brasil

\section{INTRODUÇÃO}

A audição é um dos sentidos mais nobres, uma vez que sua principal função está ligada à aquisição e ao desenvolvimento da linguagem oral, essencial nas relações interpessoais e com o meio ambiente. Esta deficiência é considerada altamente incapacitante, visto os seus efeitos na comunicação humana bem como o impacto causado no desenvolvimento cognitivo, psicossocial, na linguagem

(1) Fonoaudióloga; Mestranda em Saúde Coletiva na Faculdade de Odontologia de Bauru da Universidade de São Paulo, FOB/USP, Bauru, SP, Brasil.

(2) Fonoaudióloga; Mestranda em Saúde Coletiva na Faculdade de Odontologia de Bauru da Universidade de São Paulo, FOB/USP, Bauru, SP, Brasil.

(3) Fonoaudióloga; Professora Doutora do Departamento de Saúde Coletiva da Faculdade de Odontologia de Bauru da Universidade de São Paulo, FOB/USP, Bauru, SP, Brasil.

(4) Fonoaudióloga; Professora Doutora do Departamento de Saúde Coletiva da Faculdade de Odontologia de Bauru da Universidade de São Paulo, FOB/USP, Bauru, SP, Brasil.

Conflito de interesses: inexistente oral e escrita ${ }^{1}$. O impacto de uma privação sensorial auditiva interfere na habilidade de interpretar sons de fala e implica em desvantagens econômicas e educacionais. Pode-se observar, portanto, que a comunicação humana é de extrema importância para a constituição, desenvolvimento e aprendizado do indivíduo ${ }^{2}$.

$\mathrm{Na}$ população infantil, estudos mostraram que a detecção da deficiência auditiva é realizada tardiamente, em torno do terceiro ano de vida ${ }^{3}$. Desta forma, é importante realizar a detecção precoce desta deficiência através da triagem auditiva neonatal, procedimento capaz de detectar e intervir precocemente nas alterações auditivas que poderão interferir na vida do indivíduo. No Brasil, tais programas vêm sendo desenvolvidos desde a década de 80 com populações de alto e baixo risco, utilizando-se procedimentos comportamentais e eletrofisiológicos ${ }^{4}$.

Uma alteração que acomete a população infantil é a otite média, inflamação na orelha média frequentemente associada a um aumento de fluido infectado ou não. Sua etiologia é variada e acomete 
as vias aéreas superiores, sendo maior a prevalência na primeira infância e decrescendo com a idade. Além disso, é sabido que $50 \%$ das crianças com um ano de idade já apresentaram pelo menos um episódio de otite ${ }^{5}$, fato que pode prejudicar na aquisição e no desenvolvimento da linguagem.

Na população adulta, o impacto pode associarse ao declínio cognitivo, presença de quadros depressivos, redução do estado funcional, além de desvantagens econômicas ${ }^{6}$. As alterações auditivas adquiridas são mais comuns, sendo estas a presbiacusia e a perda auditiva induzida por ruído (PAIR). A primeira se deve ao processo de enveIhecimento. Caracteriza-se por perda auditiva, geralmente neurossensorial, bilateral, gradual e progressiva, podendo ou não apresentar zumbido associado ${ }^{7}$. Já a segunda, se deve à exposição ocupacional a intensos níveis de ruído diante a diminuição gradual da acuidade auditiva decorrente da exposição continuada a níveis elevados de pressão sonora. Tal acometimento é irreversível e quase sempre bilateral e simétrica; porém é passível de prevenção e caracteriza-se por perda auditiva neurossensorial, manifestando rebaixamento inicial nas altas frequências audiométricas ${ }^{8}$.

Neste sentido, o Ministério da Saúde estabeleceu no dia 28 de setembro de 2004, a Portaria GM no 2.073/04, a qual instituiu a Política Nacional de Atenção à Saúde Auditiva. Para o atendimento diante das ações na atenção básica, apresenta a necessidade das realizações de ações de promoção de saúde auditiva, prevenção e identificação precoce de problemas auditivos, além da organização de ações educativas e informativas, orientações e, quando necessário, encaminhamentos para a Atenção à Saúde em Média Complexidade ${ }^{9}$.

Diversos fatores são frequentemente relacionados à ocorrência de perdas auditivas, tais como idade, traumatismo craniano, exposição extraocupacional ao ruído, tabagismo, doenças sistêmicas, história familiar de déficit auditivo e exposição a agentes químicos ocupacionais ${ }^{10}$.

É dado que a epidemiologia da deficiência auditiva pode variar de forma ampla diante os fatores geográficos e econômicos, e neste sentido, a prevalência desta deficiência é menor em países cuja população tenha um padrão de vida mais alto e acesso a um sistema de saúde bem desenvolvido ${ }^{11}$.

A Fonoaudiologia, área da saúde que atende o ser humano nos problemas relativos à comunicação, a fim de conhecer melhor a população que atende, deve aliar-se à Epidemiologia, cujos estudos são essenciais para fundamentar as tomadas de decisões mais adequadas em relação à saúde da população e assim um maior conhecimento das reais necessidades da comunidade e dos fatores determinantes de agravos e doenças ${ }^{12}$. A Epidemiologia é o eixo da saúde pública ${ }^{13}$, a área do conhecimento que possibilita descrever, analisar, planejar e intervir nos problemas de saúde das coletividades humanas ${ }^{14,15}$

Desta forma, o levantamento epidemiológico da quantidade de deficientes auditivos de uma comunidade, bem como sua localização e o estudo das condições sociais são de extrema importância para a adequação das medidas de saúde pública nos vários níveis de prevenção ${ }^{16}$. Diante as limitações e consequências geradas pela deficiência auditiva na comunicação e a necessidade do entendimento da ocorrência desta deficiência nas diferentes regiões do país, esta pesquisa teve por objetivo verificar na literatura científica, estudos que realizaram levantamento das condições fonoaudiológicas relacionados à perda auditiva no Brasil.

\section{MÉTODOS}

Para o desenvolvimento do estudo epidemiológico com enfoque em Audiologia, foram realizadas buscas da literatura pertinente na seguinte base de dados e web site:- www.bireme.br e Pubmed www.pubmed.com.

Os descritores de assunto (MeSH) utilizados na base de dados foram cruzados da seguinte forma:

- Pesquisa 1: epidemiology and hearing loss and Brazil

- Pesquisa 2: (epidemiology or prevalence) and (hearing loss or deafness) and Brazil

\section{Critérios de inclusão}

Foram utilizados como critérios de inclusão os artigos publicados nos últimos 10 anos, os quais correspondem aos anos de 1999 ao ano de 2009, de qualquer idioma, bem como a inclusão de todos os artigos publicados relacionados que abordassem no título ou no resumo os descritores de acordo com a truncagem citada.

\section{Critérios de exclusão}

Os critérios de exclusão serão referentes aos artigos de revisão de literatura, artigos publicados há mais de 10 anos, que não abordassem no título ou no resumo os descritores de acordo com a truncagem citada, e que apresentassem estudos de síndromes e alterações genéticas associadas.

\section{REVISÃO DA LITERATURA E DISCUSSÃO}

Inicialmente, realizada a Pesquisa 1, foram encontrados um total de 52 artigos. Desta forma, 
optou-se por realizar a Pesquisa 2, a qual mostrouse mais completa diante o cruzamento dos descritores apresentados. Na base de dados Pubmed foram encontrados um total de 73 artigos. Aplicando-se os critérios de inclusão, chega-se a um total de 18 artigos, dentre eles, dois artigos de revisão, um não pode ser localizado e após leitura do artigo na íntegra, foi realizada a exclusão de dois artigos, visto que não condiziam com o que foi proposto pelo presente estudo. Portanto este estudo será baseado em um total de 13 artigos, de acordo com a Figura 1.

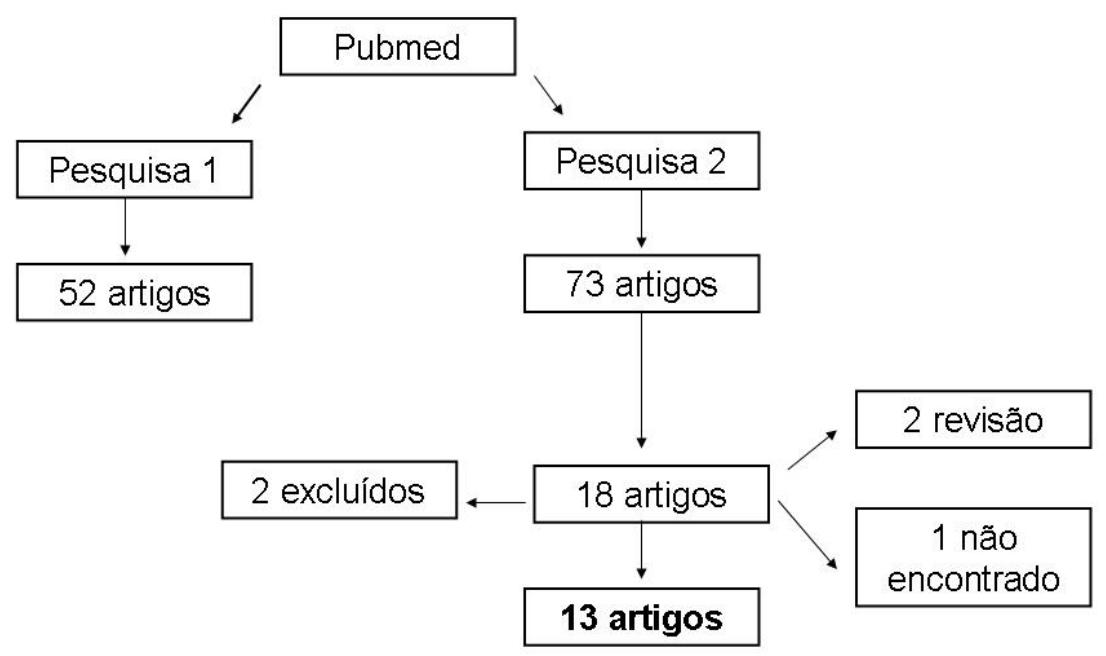

Figura 1 - Organograma da busca realizada

A faixa etária estudada por estes trabalhos variam desde neonatos a idosos. Dentre os tipos de estudos presentes, foi encontrado o estudo transversal em 11 artigos, o estudo caso-controle em um artigo, e o estudo de coorte em outro artigo; o que corresponde a $84,62 \%, 7,69 \%, 7,69 \%$, do total de artigos, respectivamente. A Tabela 1 apresenta os artigos utilizados neste estudo.

No presente estudo observa-se a presença de três pesquisas que buscaram estudar a presença de perda auditiva em crianças, dentre elas escolares e neonatos. Destas, uma pesquisa ${ }^{6}$ deu enfoque aos neonatos, buscando relacionar os fatores de risco para deficiência auditiva em nascidos no Hospital público de Belo Horizonte, Minas Gerais através de um estudo transversal.

Com base nos registros do Programa de Triagem Auditiva, foram analisados dados de 789 crianças. A prevalência da deficiência auditiva foi de $1,8 \%$, e foi observada associação estatisticamente significativa entre os fatores de risco como: suspeita de surdez por parte dos familiares, hiperbilirrubinemia, medicação ototóxica, baixo peso ao nascer (menor que $1.500 \mathrm{~g}$ ). Mattos et al (2007) ${ }^{17}$ relataram que a detecção e o tratamento precoce trará amplos benefícios quanto ao desenvolvimento social e comunicativo da criança, seja com a utilização de amplificação sonora individual ou com o implante coclear, sobretudo quando realizado antes dos seis meses de idade.

Piatto e Meniglia (2001) ${ }^{18}$ em sua pesquisa analisaram a prevalência de perda auditiva em crianças de 3 a 6 anos de idade em creches municipais. A amostra foi constituída por 103 crianças, sendo que destas, 9,7\% apresentaram alterações audiométricas. Já Godinho et al. (2001) ${ }^{19}$ investigaram a prevalência de alterações otológicas em 1.005 crianças e adolescentes na faixa etária de 6 a 18 anos, de escolas públicas e particulares do município de Belo Horizonte. Os autores verificaram que a prevalência de otite média crônica em $0,94 \%$, de rolha de cera, $12,3 \%$, e em $10,5 \%$ apresentava outras alterações. Devido a fatores intrínsecos no desenvolvimento da linguagem e de prejuízos sociais, emocionais e cognitivos ${ }^{6}$, faz-se necessária a realização da avaliação auditiva na faixa etária escolar. Por outro lado, esses estudos, por avaliarem somente as crianças inseridas no ambiente escolar não permitem determinar ou extrapolar taxas de prevalências para a população, em igual faixa etária, pois se trata de uma determinada área ou, ainda, que podem estar distorcidos pela evasão escolar existente no Brasil, que atinge justamente crianças e adolescentes mais carentes ${ }^{16}$.

A análise das pesquisas encontradas neste estudo pode mostrar que seis artigos buscaram 
Tabela 1 - Artigos utilizados no presente estudo de acordo com autor(es), ano de publicação, localização, sujeitos do estudo e resultados

\begin{tabular}{|c|c|c|c|c|c|}
\hline Autor & $\begin{array}{c}\text { Ano de } \\
\text { publicação }\end{array}$ & $\begin{array}{c}\text { Localização no } \\
\text { Brasil }\end{array}$ & $\begin{array}{c}\text { Sujeitos dos } \\
\text { estudos/N }\end{array}$ & Objetivos & Resultados (\%) \\
\hline $\begin{array}{l}\text { Piatto e } \\
\text { Meniglia }\end{array}$ & 2001 & $\begin{array}{c}\text { São José do Rio } \\
\text { Preto/SP }\end{array}$ & Crianças/ 103 & $\begin{array}{c}\text { Prevalência de perda } \\
\text { auditiva }\end{array}$ & $9,7 \%$ PA \\
\hline Godinho et al. & 2001 & $\begin{array}{c}\text { Belo } \\
\text { Horizonte/MG }\end{array}$ & $\begin{array}{c}\text { Crianças e } \\
\text { adolescentes/ } \\
1.005\end{array}$ & $\begin{array}{c}\text { Investigar a } \\
\text { prevalência de } \\
\text { alterações otológicas }\end{array}$ & $\begin{array}{c}\text { 0,94\% OMC } \\
12,3 \% \text { Cerumen }\end{array}$ \\
\hline $\begin{array}{l}\text { Corrêa-Filho } \\
\text { et al. }\end{array}$ & 2002 & Campinas/SP & $\begin{array}{c}\text { Adultos - } \\
\text { Trabalhadores/ } \\
108\end{array}$ & $\begin{array}{c}\text { Prevalências de } \\
\text { perda auditiva } \\
\text { induzida por ruído e } \\
\text { hipertensão arterial } \\
\text { em condutores de } \\
\text { ônibus urbanos }\end{array}$ & $32,7 \%$ PAIR \\
\hline $\begin{array}{c}\text { Harger e } \\
\text { Barbosa- } \\
\text { Branco }\end{array}$ & 2004 & Brasília/DF & $\begin{array}{c}\text { Adultos - } \\
\text { Trabalhadores/ } \\
152\end{array}$ & $\begin{array}{c}\text { Prevalência de } \\
\text { perdas auditivas, em } \\
\text { trabalhadores de } \\
\text { marmorarias }\end{array}$ & $48 \%$ PAIR \\
\hline $\begin{array}{c}\text { Barbosa e } \\
\text { Cardoso }\end{array}$ & 2005 & São Paulo/SP & $\begin{array}{c}\text { Adultos - } \\
\text { Trabalhadores/ } \\
624\end{array}$ & $\begin{array}{l}\text { Avaliação auditiva de } \\
\text { trabalhadores } \\
\text { expostos ao ruído do } \\
\text { tráfego urbano }\end{array}$ & $28,5 \%$ PAIR \\
\hline Guerra et al. & 2005 & $\begin{array}{c}\text { Rio de } \\
\text { Janeiro/RJ }\end{array}$ & $\begin{array}{c}\text { Adultos - } \\
\text { Trabalhadores/ } \\
182\end{array}$ & $\begin{array}{c}\text { Prevalência de casos } \\
\text { sugestivos de PAIR } \\
\text { em trabalhadores } \\
\text { metalúrgicos }\end{array}$ & $15,9 \%$ PAIR \\
\hline Béria et al. & 2007 & Canoas/RS & $\begin{array}{c}\text { Populacional/ } \\
2.427\end{array}$ & $\begin{array}{c}\text { Determinar } \\
\text { alterações de orelha } \\
\text { e audição em uma } \\
\text { base populacional }\end{array}$ & $\begin{array}{l}26,1 \% \text { PA e } 6,8 \% \\
\text { PA incapacitante }\end{array}$ \\
\hline Tiensoli et al. & 2007 & $\begin{array}{c}\text { Belo } \\
\text { Horizonte/MG }\end{array}$ & $\begin{array}{c}\text { Neonatos e } \\
\text { lactentes/ } \\
798\end{array}$ & $\begin{array}{c}\text { Relacionar fatores de } \\
\text { risco com a } \\
\text { deficiência auditiva }\end{array}$ & $\begin{array}{l}\text { 1,8\% de perda } \\
\text { auditiva e } \\
\text { significância } \\
\text { entre os fatores } \\
\text { de risco }\end{array}$ \\
\hline El Dib et al. & 2008 & São Paulo/SP & $\begin{array}{c}\text { Adultos - } \\
\text { Trabalhadores/ } \\
177\end{array}$ & $\begin{array}{l}\text { Prevalência de perda } \\
\text { auditiva em altas } \\
\text { freqüências na } \\
\text { exposição ao ruído } \\
\text { entre técnicos e } \\
\text { controladores de som }\end{array}$ & $\begin{array}{c}50 \% \text { e } 10,5 \% \\
\text { respectivamente } \\
\text { apresentando } \\
\text { PAIR }\end{array}$ \\
\hline Ogido et al. & 2009 & Campinas/SP & $\begin{array}{c}\text { Adultos - } \\
\text { Trabalhadores/ } \\
175\end{array}$ & $\begin{array}{c}\text { Prevalência de } \\
\text { sintomas auditivos e } \\
\text { vestibulares em } \\
\text { trabalhadores } \\
\text { expostos a ruído } \\
\text { ocupacional/ } 175\end{array}$ & $\begin{array}{c}74 \% \text { PA, } 81 \% \\
\text { zumbido e } \\
13,2 \% \text { vertigem }\end{array}$ \\
\hline $\begin{array}{l}\text { Batissoco } \\
\text { et al. }\end{array}$ & 2009 & São Paulo/SP & $\begin{array}{c}\text { Crianças e adultos/ } \\
300\end{array}$ & $\begin{array}{c}\text { Estabelecer a } \\
\text { frequência de } \\
\text { mutações da proteína } \\
\text { GJB2 e deleções do } \\
\text { GJB6 }\end{array}$ & $\begin{array}{c}12,4 \% \text { e } 1 \% \\
\text { respectivamente }\end{array}$ \\
\hline Piltcher et al. & 2000 & Porto Alegre/RS & $\begin{array}{c}\text { Crianças e adultos/ } \\
28\end{array}$ & $\begin{array}{c}\text { Prevalência de perda } \\
\text { auditiva } \\
\text { sensorioneural }\end{array}$ & $21,4 \% \mathrm{PA}$ \\
\hline Cruz et al. & 2009 & São Paulo/SP & $\begin{array}{c}\text { Populacional/ } \\
5.250\end{array}$ & $\begin{array}{l}\text { Prevalência de } \\
\text { deficiência auditiva } \\
\text { referida em quatro } \\
\text { localidades do } \\
\text { Estado de SP }\end{array}$ & $5,21 \% \mathrm{PA}$ \\
\hline
\end{tabular}


estudar o impacto do ruído ocupacional na audição do trabalhador 10,20-24. Além disso, observaram a presença de zumbido como queixa de alguns sujeitos de pesquisa, fato que de acordo com estudo realizado por Amorim et al. (2008) ${ }^{25}$, é um dos sintomas mais frequentes, seguido por dificuldade na compreensão de fala em ambientes ruidosos. Tais estudos foram realizados com diferentes profissionais como metalúrgicos ${ }^{10}$, condutores de ônibus ${ }^{22,24}$, técnicos de som ${ }^{21}$, o que corrobora os estudos de Miranda et al (1998) ${ }^{26}$ e Russo ICP (1995) ${ }^{27}$, além disso foi observada a prevalência de indivíduos adultos do gênero masculino. Importante salientar que nestes casos faz-se necessária a realização de estudos associados ao uso dos equipamentos de proteção individual (EPI), visto que é uma das formas de prevenção da PAIR, utilizado contra possíveis riscos ameaçadores da saúde auditiva ${ }^{28}$.

Outro estudo observou prevalências menores de casos sugestivos de PAIR em trabalhadores que relataram regularidade do uso, em comparação com os que informaram uso irregular ou não uso 10. Estudos devem ser conduzidos com o objetivo de se relacionar a perda auditiva e o ambiente de trabalho, tanto pelo crescimento e desenvolvimento industrial que o país vivencia, quanto para promover o bem-estar e melhoria da qualidade de vida da população trabalhista deste país.

De acordo com a Política Nacional de Saúde do Portador de Deficiência é apontada como a principal causa de perda de audição entre adultos ${ }^{17}$. No presente estudo não foram encontradas pesquisas que, especificadamente, buscassem obter a prevalência da perda auditiva na população idosa, porém dentre os estudos ${ }^{10,29}$ encontrados, alguns abrangiam em sua faixa etária a população idosa. Alguns autores relataram a existência de fatores intrínsecos e extrínsecos relacionados à presbiacusia, a qual então não seria apenas devido ao aumento da idade do indivíduo ${ }^{30}$. Tal fato pode ser observado diante a presença de estudos relacionados à PAIR ${ }^{10}$ que mostraram a presença da população idosa como sujeitos de pesquisa, e outro estudo que relata a presença de declínio da acuidade auditiva, relacionada ao tempo de exposição ao ruído ocupacional ${ }^{20}$.

Na busca realizada, pode-se notar o interesse de alguns pesquisadores em conhecer a prevalência de perda auditiva por meio do auxílio de técnicas laboratoriais ${ }^{31,32}$, o que pode vir a enriquecer os estudos relativos às perda auditivas idiopáticas e fornecer informações para uma intervenção precoce. Nota-se também o interesse por parte de pesquisadores no conhecimento da prevalência da deficiência auditiva referida pela população, sendo este por meio de um questionário com respostas em conjunto fechado, além da associação de fatores que podem ser associados como diabetes, hipertensão, acidente vascular encefálico, uso de medicamentos, e depressão ${ }^{16}$. De acordo com os autores do trabalho, este é de grande valia visto que $o$ inquérito populacional pode ser realizado por profissionais de diferentes áreas de atuação e oferece assim maior precisão quanto à resolubilidade de problemas, sendo uma ferramenta para auxiliar na formulação das políticas públicas de saúde.

Por fim, uma pesquisa buscou estudar a prevalência de perda auditiva no Sul do Brasil, abrangendo uma população de indivíduos com idade a partir de quatro anos, seguindo o Protocolo de avaliação da Prevalência de Surdez e outros Transtornos de Audição ${ }^{33}$. Este foi o primeiro estudo realizado na América Latina seguindo tal Protocolo, e seus principais objetivos foram os de aumentar a conscientização, determinar prioridades, selecionar estratégias de prevenção, prever necessidades, auxiliar a medir a carga da doença e a análise econômica ${ }^{29}$. Mais estudos são necessários por apresentarem-se como um meio de buscar a comparação real através de alguns estudos padronizados e assim direcionar as ações referentes à política de saúde auditiva do país, diante as diversidades geográficas, socioeconômicas e culturais vigentes.

Por essa razão, sugere-se que estudos de abrangência nacional, analisando a deficiência auditiva, devam ser realizados, para que cada abordagem seja contextualizada frente às questões sócio-econômicas locais, e desta forma, ações de saúde e assistência possam ser adequadas às diferentes regiões do país.

\section{CONCLUSÃO}

Após a avaliação e a análise dos artigos incluídos neste estudo, concluiu-se que está ocorrendo maior preocupação em relação às alterações auditivas ocorridas devido à atividade laboral. Outros grupos não estão sendo tão contemplados nos estudos científicos, como a população idosa e neonatal. A identificação precoce da deficiência auditiva permitiria a redução dos custos para os serviços públicos, além de melhorar a qualidade de vida do indivíduo. 


\section{ABSTRACT}

Background: hearing is responsible for acquisition and development of oral language, this sense allows for interpersonal and environmental relationship. Epidemiological investigations on hearing impairment in a community are very important in order to adequate the public health measures at different prevention levels. Purpose: to check, in the scientific literature, studies that aimed at the pursuit of hearing loss epidemiology knowledge in Brazil. Thirteen articles were used in this study and 11 were a cross-sectional studies, one a case-control study and another a cohort study. Conclusion: Workers exposed to occupational noise have received more attention from epidemiological studies than the elderly and neonate population. Just one population-based study was carried out following the protocol suggested by WHO. It is therefore important to carry out further studies related to hearing loss in the country in order to develop health care and assistance actions that are appropriate to the local needs.

KEYWORDS: Audiology; Epidemiology; Hearing Loss; Brazil

\section{REFERÊNCIAS}

1. Freitas CD, Costa MJ. Processo de adaptação de próteses auditivas em usuários atendidos em uma instituição pública federal - parte I: resultados e implicações com o uso da amplificação. Rev Bras Otorrinolaringol. 2007; 73(6):744-51.

2. Horacio CP, Goffi-Gomez MVS. A contribuição da leitura orofacial na comunicação do neuropata auditivo. Rev. CEFAC. 2007; 9(3):404-10.

3. Durante AS, Carvalho RMM, Costa MTZ, Cianciarullo MA, Voegels RL, Takahashi GM, et al. A implementação de programa de triagem auditiva neonatal universal em um hospital universitário brasileiro. Pediatria. 2004; 26(2):78-84.

4. Castro Junior NPC, Marona SAM, Almeira CIR, Redondo MC. Avaliação audiológica em recém-nascidos. In: Campos $\mathrm{CAH}$, Costa HOO, organizadores. Tratado de otorrinolaringologia. São Paulo: Roca; 2002. p. 441-51.

5. Wertzner HF, Pagan LO, Galea DES, Papp ACCS. Características fonológicas de crianças com transtorno fonológico com e sem histórico de otite média. Rev Soc Bras Fonoaudiol. 2007; 12(1):41-7. 6. Tiensoli LO, Goulart LMHF, Resende LM, Colosimo EA. Triagem auditiva em hospital público de Belo Horizonte, Minas Gerais, Brasil: deficiência auditiva e seus fatores de risco em neonatos e lactentes. Cad. Saúde Pública. 2007; 23(6):1431-41. 7. Jerger $S$, Jerger J. Alterações auditivas: um manual para avaliação clínica. São Paulo: Atheneu; 1998.

8. Nudelmann AA, Costa EA, Seligman J, Ibañez RN. Perda auditiva induzida pelo ruído. Porto Alegre: Bagaggem; 1997.

9. Brasil. Ministério da Saúde. Saúde auditiva. Portarias de saúde auditiva. Brasília, DF: Ministério da Saúde; 2004 [acesso em 2009 set 25]. Disponível em: http://portal.saude.gov.br/portal/sas/mac/area. cfm?id_area $=848$.

10. Guerra MR, Lourenço PMC, BustamanteTeixeira MT, Alves MJM. Prevalência de perda auditiva induzida por ruído em empresa metalúrgica. Rev Saúde Pública. 2005; 39(2):238-44.

11. Russo, ICP. A relevância da pesquisa científica na audiologia brasileira. Rev. CEFAC. 2009; 11(Supl1):1-4.

12. Lima BPS, Guimarães JATL, Rocha MCG. Características epidemiológicas das alterações de linguagem em um centro fonoaudiológico do primeiro setor. Rev Soc Bras Fonoaudiol. 2008; 13(4):376-80.

13. Rouquayrol MZ, Filho NA. Epidemiologia e saúde. Rio de Janeiro: Medsi; 2003.

14. Bergonzoli G. Epidemiology and genetics: a strategic alliance in the new millennium? Rev Panam Salud Pública. 2005; 17(1):38-45.

15. Lessa F. Fonoaudiologia e epidemiologia. In: Ferreira L, Befi-Lopes DM, Limongi SCO, organizadores. Tratado de fonoaudiologia. São Paulo: Roca; 2004. p. 527-37.

16. Cruz MS, Oliveira LR, Carandina L, Lima MCP, César CLG, Barros MBA et al. Prevalência de deficiência auditiva referida e causas atribuídas: um estudo de base populacional. Cad Saúde Pública. 2009; 25(5):1123-31.

17. Matos LC, Veras RP. A prevalência da perda auditiva em uma população de idosos da cidade do Rio de Janeiro: um estudo seccional. Rev Bras Otorrinolaringol. 2007; 73(5):654-9.

18. Piatto VB, Meniglia JV. Hearing evaluation in children aged 3-6 years in day-care centers. J Pediatr. 2001; 77(2):124-30. 
19. Godinho RN, Gonçalves TML, Nunes FB, Becker CG, Becker HMG, Guimarães RES, et al. Prevalence and impact of chronic otitis media in school age children in Brazil. First epidemiologic study concerning chronic otitis media in Latin America. Int J Pediatr Otorhinolaryngol. 2001; 61:223-32.

20. Ogido R, Costa EA, Machado HC. Prevalência de sintomas auditivos e vestibulares em trabalhadores expostos a ruído ocupacional. Rev Saúde Pública. 2009; 43(2):377-80.

21. El Dib RP, Silva EMK, Morais JF, Trevisani VFM. Prevalence of high frequency hearing loss consistent with noise exposure among people working with sound systems and general population in Brazil: a cross-sectional study. BMC Public Health. 2008; 8:151.

22. Barbosa ASM, Cardoso MRA. Hearing loss among workers exposed to road traffic noise in the city of São Paulo in Brazil. Auris Nasus Larynx. 2005; 32:17-21.

23. Harger MRHC, Barbosa-Branco A. Efeitos auditivos decorrentes da exposição ocupacional ao ruído em trabalhadores de marmorarias no Distrito Federal. Rev Assoc Med Bras. 2004; 50(4):396-9.

24. Corrêa Filho HR, Costa LC, Hoehne EL, Pérez MAG, Nacimento LCR, Moura EC. Perda auditiva induzida por ruído e hipertensão em condutores de ônibus. Rev Saúde Pública. 2002; 36(6):693-701.

25. Amorim RB, Lopes AC, Santos KTP, Melo ADP, Lauris JRP. Alterações auditivas da exposição ocupacional em músicos. Arq int otorrinolaringol. 2008; 12(3):377-83.

26. Miranda CR, Dias CR, Pena PGL, Nobre LCC, Aquino R. Perda auditiva induzida pelo ruído em trabalhadores industriais da região metropolitana de Salvador, Bahia. Rev Bras Otorrinolaringol. 1998; 64:109-14.

27. Russo ICP, Santos TMM, Busgaib BB, Osterne FJV. Um estudo comparativo sobre os efeitos da exposição à música em músicos de trio elétrico. Rev Bras Otorrinolaringol. 1995; 61:477-84.

28. Santos JD, Ferreira MIDC. Variação dos limiares audiométricos em trabalhadores submetidos a ruído ocupacional. Arq Int Otorrinolaringol. 2008; 12(2):201-19.

29. Béria JU, Raymann BCW, Gigante LP, Figueiredo ACL, Jotz G, Roithman R, et al. Hearing impairment and socioeconomic factors: a population-based survey of an urban locality in southern Brazil. Rev Panam Salud Publica. 2007; 21(6):381-7

30. Gates GA, Cooper JC, William BK, Miller NJ. Hearing in the elderly: the Framingham Cohort, 1983-1985. Ear Hear. 1990; 11(Pt 1):247-56.

31. Batissoco AC, Abreu-Silva RS, Braga MCC, Lezirovitz K, Della-Rosa V, Tabith Junior A, et al. Prevalence of GJB2 (Connexin-26) and GJB6 (Connexin-30) Mutations in a Cohort of 300 Brazilian Hearing-Impaired Individuals: Implications for Diagnosis and Genetic Counseling. Ear Hear. 2009; 30(1):1-7.

32. Piltcher O, Cigana L, Friedriech J, Ribeiro FA, da Costa SS. Sensorineural hearing loss among sickle cell disease patients from southern Brazil. Am J Otolaryngol. 2000; 21(2):75-9.

33. WHO. Ear and hearing disorders survey. Protocol for a population-based survey of prevalence and causes of deafness and hearing impairment and other ear diseases. Prevention of Blindness and Deafness (PBD). Geneva: WHO; 1999.

DOI: 10.1590/S1516-18462010005000089

RECEBIDO EM: 21/01/2010

ACEITO EM: 01/04/2010

Endereço para correspondência:

Aline Megumi Arakawa

Al. Dr. Octávio Pinheiro Brisolla, 9-75

Bauru - SP

CEP: 17012-901

E-mail: alinearakawa@usp.br 